\title{
AT9283 exhibits antiproliferative effect on tyrosine kinase inhibitor-sensitive and -resistant chronic myeloid leukemia cells by inhibition of Aurora A and Aurora B
}

\author{
TOMOYA TAKEDA $^{1}$, MASANOBU TSUBAKI ${ }^{1}$, SHUJI GENNO $^{1}$, CHISATO NEMOTO $^{1}$, YASUKA ONISHI ${ }^{1}$, \\ YUUTA YAMAMOTO $^{1}$, MOTOHIRO IMANO ${ }^{2}$, TAKAO SATOU ${ }^{3}$ and SHOZO NISHIDA $^{1}$ \\ ${ }^{1}$ Division of Pharmacotherapy, Kindai University School of Pharmacy, Higashi-Osaka, Osaka 577-8502; \\ Departments of ${ }^{2}$ Surgery and ${ }^{3}$ Pathology, Kindai University School of Medicine, Osakasayama, Osaka 589-0014, Japan
}

Received March 21, 2020; Accepted July 13, 2020

DOI: $10.3892 / o r .2020 .7739$

\begin{abstract}
Imatinib is the gold standard in the conventional treatment of chronic myeloid leukemia (CML). However, some patients become resistant to imatinib therapy. To overcome this resistance, second-generation (dasatinib, nilotinib, and bosutinib) and third-generation (ponatinib) tyrosine kinase inhibitors (TKIs) have been developed and have been shown to be effective against refractory CML. Although these TKIs provide many benefits for patients with CML, advanced patients show resistance even to these TKIs. Therefore, novel therapeutic strategies are urgently needed for the treatment of TKI-resistant CML patients. AT9283 is a multi-targeted kinase inhibitor with potent activity against Janus kinase (JAK), Aurora kinases, and Abl. In the present study, we showed that AT9283 significantly decreased the cell viability of both TKI-sensitive and TKI-resistant CML cells as determined by trypan blue exclusion assay. In addition, cell cycle analysis, Annexin V assay, and caspase-3/7 activity assay revealed that AT9283 increased the cell population in the $\mathrm{G} 2 / \mathrm{M}$ phase and induced apoptosis. We investigated the molecular mechanisms underlying the decrease in cell viability upon treatment with AT9283 by western blotting. Interestingly, our results showed that AT9283 inhibited the expression of Aurora A, Aurora B, and downstream Histone $\mathrm{H} 3$ phosphorylation. In contrast, we observed no changes in the levels of Bcr-Abl, signal transducer and activator of transcription 3 (STAT3), extracellular signal-regulated kinase (ERK), and Akt
\end{abstract}

Correspondence to: Dr Shozo Nishida, Division of Pharmacotherapy, Kindai University School of Pharmacy, 3-4-1 Kowakae, Higashi-Osaka, Osaka 577-8502, Japan

E-mail: nishida@phar.kindai.ac.jp

Abbreviations: CML, chronic myeloid leukemia; TKI, tyrosine kinase inhibitor; K562/IR, imatinib-resistant K562 cells; JAK, Janus kinase; ERK1/2, extracellular signal-regulated kinase 1/2; STAT3, signal transducer and activator of transcription 3

Key words: chronic myeloid leukemia, AT9283, Aurora A, Aurora B, imatinib phosphorylation. In addition, we found that AMG900, a selective Aurora A and Aurora B inhibitor, increased the G2/M phase cell population and induced apoptosis via inhibition of Aurora $A$ and Aurora B in both TKI-sensitive and TKI-resistant CML cells. Our studies show that Aurora A and Aurora B are promising therapeutic targets for TKI-sensitive and TKI-resistant CML, and AT9283 may have potential clinical applications for the treatment of TKI-resistant CML patients.

\section{Introduction}

Chronic myeloid leukemia (CML), a hematological malignancy, occurs as a result of the presence of the Bcr-Abl fusion protein (1). The Bcr-Abl fusion protein constitutively activates tyrosine kinase activity and induces many downstream signaling pathways, such as phosphoinositide 3 kinase (PI3K)/Akt, Janus kinase (JAK)/signal transducer and activator of transcription (STAT) and mitogen-activated protein kinase (MAPK)/extracellular signal-regulated kinase (ERK) pathways, contributing to cell survival and proliferation $(2,3)$. Imatinib, a tyrosine kinase inhibitor (TKI) that binds to the ATP-binding site of ABL, has dramatically advanced the treatment of CML by inducing long-term overall survival rates of $>90 \%$ (4). However, approximately $35 \%$ of patients develop pharmacological resistance to imatinib (5). To overcome this resistance, second-generation (dasatinib, nilotinib, and bosutinib) and third-generation (ponatinib) TKIs have been developed and have been shown to be effective against refractory CML patients $(6,7)$. Although these drugs provide many benefits for patients with CML, advanced patients show resistance even to these TKIs (8). Therefore, novel therapeutic strategies are urgently needed for the treatment of TKI-resistant CML patients.

The Aurora family of serine/threonine kinases is essential for many cellular functions, including centrosome function, spindle assembly, chromosome segregation, and cytokinesis $(9,10)$. They comprise Aurora A and Aurora B, and elevated expression of Aurora A and Aurora B have been shown in human cancers such as breast, ovarian, gastric, colon, and pancreatic cancers $(11,12)$. Furthermore, inhibition of Aurora A and Aurora B has proven to be an attractive anticancer approach for a variety of cancers including solid tumors and hematological malignancies (13-15). 
Therefore, inhibiting Aurora kinase is a potential target for the treatment of CML. JAK has long been recognized to be involved in CML pathogenesis (16). JAKs are cytoplasmic protein tyrosine kinases, and dysregulation of the JAK/STAT pathways is critically involved in the survival and proliferation of CML cells (17). Therefore, blocking the JAK/STAT pathway may also represent a novel therapeutic strategy for CML patients.

AT9283 is a multi-targeted kinase inhibitor, discovered using a fragment-based approach, with potent activity against JAK, Aurora kinases, and Abl (18). Previous studies have shown that AT9283 has therapeutic potential in leukemic cells, myeloproliferative disorders, and colon cells (19). In our previous study, the K562/IR cell line (imatinib-resistant CML cells) was established, and K562/IR exhibited resistance to TKIs including imatinib, nilotinib, dasatinib, bafetinib, and ponatinib (20). Here, we showed that AT9283 significantly decreased the cell viability of both TKI-sensitive and TKI-resistant CML cells, including K562/IR. Furthermore, we investigated the molecular mechanisms underlying the decrease in the cell viability. Importantly, we found the critical role of Aurora A and Aurora B in the antiproliferative effects of AT9283 in both TKI-sensitive and TKI-resistant CML cells. Our studies suggest that Aurora A and Aurora B are promising therapeutic targets in TKI-sensitive and TKI-resistant CML, and AT9283 may have potential clinical applications for CML treatment.

\section{Materials and methods}

Materials. AT9283 and AMG900 (Adooq Bioscience) were first dissolved in dimethyl sulfoxide (DMSO) to a concentration of $50 \mathrm{mM}$ (stock solution) and stored at $-20^{\circ} \mathrm{C}$. These reagents were diluted in phosphate-buffered saline (PBS) before use in the experiments described below.

Cells and culture conditions. The human CML cell line K562 was obtained from the Health Science Research Resources Bank (Osaka, Japan). K562/IR cells were obtained from our laboratory. These cells were maintained in RPMI-1640 medium (Sigma-Aldrich; Merck KGaA) with $10 \%$ fetal bovine serum (FBS) (Gibco; Thermo Fisher Scientific, Inc.), 2 mM L-glutamine (Wako Pure Chemical Industries, Ltd.), 25 mM HEPES (Wako Pure Chemical Industries, Ltd.), $100 \mu \mathrm{g} / \mathrm{ml}$ penicillin (Gibco; Thermo Fisher Scientific, Inc.), and $100 \mathrm{U} / \mathrm{ml}$ streptomycin (Gibco; Thermo Fisher Scientific, Inc.) at $37^{\circ} \mathrm{C}$ in $5 \% \mathrm{CO}_{2}$.

Trypan blue exclusion assay. CML cells were plated in 96-well plates at a concentration of $2 \times 10^{4}$ cells $/ \mathrm{ml}$. Then, AT9283 $(10,30,50$, and $100 \mathrm{nM})$ or AMG900 $(10,50,100,300$, and $500 \mathrm{nM})$ were added to the well. After 3 days, CML cells were stained with trypan blue and the number of stained cells was counted at a magnification of x100 using an Olympus CK2 inverted microscope (Olympus Optical Co.).

Cell cycle analysis. Assessment of the cell cycle analysis was performed with the Muse ${ }^{\mathrm{TM}}$ cell cycle kit (Merck Millipore), according to the manufacturer's protocol. CML cells were treated with 50 nM AT9283 or 300 nM AMG900 for 2 days. Then, Muse ${ }^{\text {TM }}$ cell cycle reagent was added and incubated at room temperature for $30 \mathrm{~min}$ in the dark. After incubation, the cell cycle analysis was performed using a Muse Cell Analyzer (Merck Millipore).
Annexin V assay. Measurement of cells undergoing apoptosis was performed with the Muse ${ }^{\mathrm{TM}}$ Annexin-V and Dead Cell Assay kit (Merck Millipore), according to the manufacturer's protocol. CML cells were treated with $50 \mathrm{nM}$ AT9283 or $300 \mathrm{nM}$ AMG900 for 2 days. Then, Muse ${ }^{\mathrm{TM}}$ Annexin V and dead cell reagent were added. After incubation for $20 \mathrm{~min}$ at room temperature, apoptotic cells were applied to a Muse Cell Analyzer (Merck Millipore).

Caspase-3/7 activity assay. Measurement of cells undergoing caspase-3/7 activation was performed using the Muse ${ }^{\mathrm{TM}}$ Caspase-3/7 kit (Merck Millipore), according to the manufacturer's protocol. CML cells were treated with $50 \mathrm{nM}$ AT9283 or 300 nM AMG900 for 2 days. Then, Muse ${ }^{\mathrm{TM}}$ Caspase- $3 / 7$ reagent was added and incubated at $37^{\circ} \mathrm{C}$ for $30 \mathrm{~min}$. After incubation, Muse $^{\mathrm{TM}}$ Caspase 3/7-AAD reagent was added and incubated at room temperature for $5 \mathrm{~min}$. The activation of caspase-3/7 was applied to a Muse Cell Analyzer (Merck Millipore).

Western blotting. CML cells were cultured with different concentrations of AT9283 or AMG900 and lysed using lysis buffer as previously described (21). The protein concentration of the lysate was measured using the BCA protein assay kit (Thermo Fisher Scientific, Inc.). The extracts $(40 \mu \mathrm{g})$ were separated by $10 \%$ sodium dodecyl sulfate-polyacrylamide gel electrophoresis (SDS-PAGE) and transferred to polyvinylidene fluoride (PVDF) membranes (GE Healthcare). The membranes were blocked in TBS plus 5\% skim milk and incubated overnight at $4^{\circ} \mathrm{C}$ with each of the following antibodies: Anti-phospho-Abl (cat. no. 2865; dilution 1:1,000), anti-Abl (cat. no. 2862; dilution 1:1,000), anti-phospho-STAT3 (cat. no. 9131; dilution 1:1,000), anti-STAT3 (cat.no.9132; dilution 1:1,000), anti-phospho-ERK1/2 (cat. no. 4370; dilution 1:2,000), anti-ERK1/2 (cat. no. 9102; dilution 1:2,000), anti-phospho-Akt (cat. no. 9271; dilution 1:1,000), anti-Akt (cat. no. 9272; dilution 1:1,000), anti-phospho-Aurora A (cat. no. 2914; dilution 1:1,000), anti-Aurora A (cat. no. 4718; dilution 1:1,000), anti-phospho-Aurora B (cat. no. 2914; dilution 1:1,000), anti-Aurora B (cat. no. 3094; dilution 1:1,000), anti-phospho-Histone H3 (cat. no. 3377; dilution 1:1,000), anti-Histone H3 (cat. no. 4499; dilution 1:1,000; all from Cell Signaling Technology, Inc.), anti- $\beta$-actin (product no. A2228; dilution 1:3,000; Sigma-Aldrich; Merck KGaA), and anti-Lamin A/C (cat. no. sc-20681; dilution 1:1,000; Santa Cruz Biotechnologies, Inc.). Subsequently, the membranes were incubated with anti-rabbit (cat. no. 7074; dilution 1:5,000; Cell Signaling Technology, Inc.) or anti-mouse (cat. no. 7076; dilution 1:5,000; Cell Signaling Technology, Inc.) secondary antibody. The proteins were visualized using Luminata Forte Western HRP substrate (Merck Millipore) according to the manufacturer's instructions. The quantities of reactive proteins were determined based on densitometric measurements using a CS analyzer (ATTO, Tokyo, Japan).

Statistical analysis. All results are expressed as the mean \pm standard deviation (SD) of three independent experiments. All analyses were conducted using SPSS version 21.0 software (IBM Corp.), and Shapiro-Wilk analysis and one-way analysis of variance (ANOVA) followed by Dunnett's test. P-value $<0.05$ was considered as indicative of a statistically significant result. 

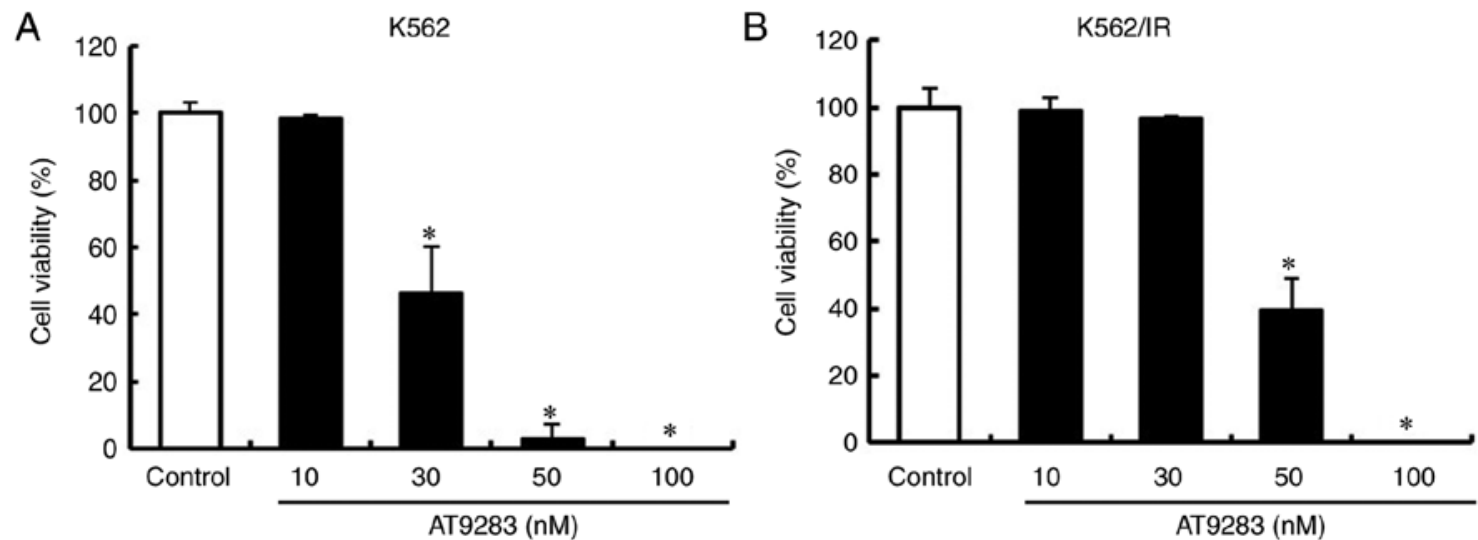

Figure 1. AT9283 decreases the cell viability of TKI-sensitive and TKI-resistant CML cells. (A) K562 cells and (B) K562/IR cells were treated with AT9283 $(10,30,50$, and $100 \mathrm{nM})$. Trypan blue exclusion assay was performed on the cells after 3 days. The results are expressed as the mean \pm SD of 3 experiments performed in triplicate. ${ }^{*} \mathrm{P}<0.05$ compared to the control. TKI, tyrosine kinase inhibitor; CML, chronic myeloid leukemia.

A
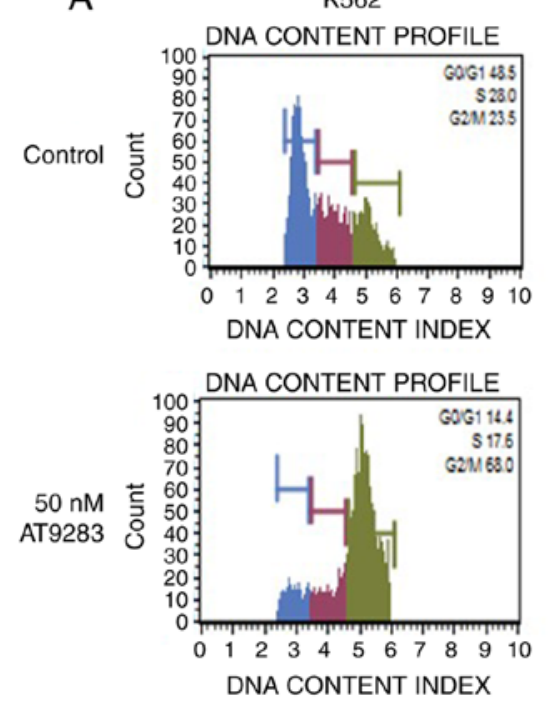

B

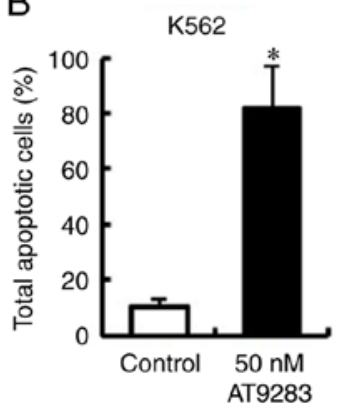

$\mathrm{K} 562 / \mathrm{IR}$

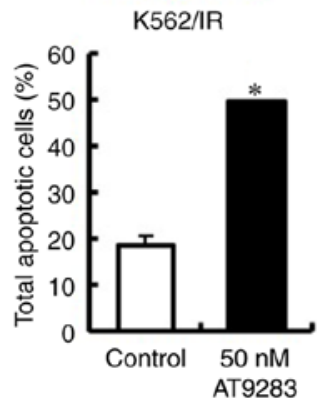

K562/IR
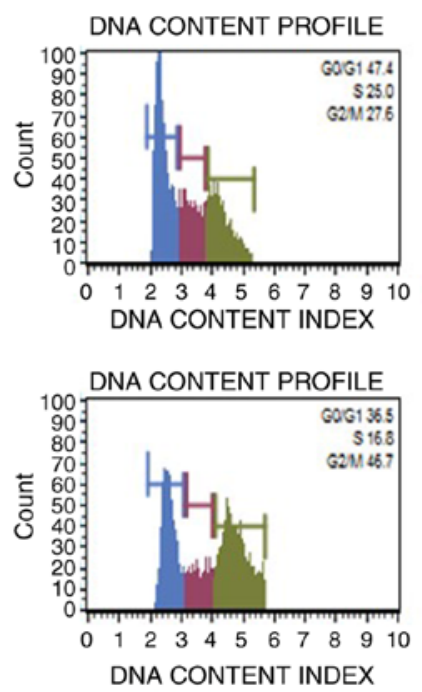

C

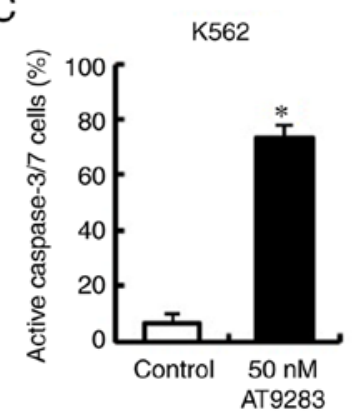

K562/IR

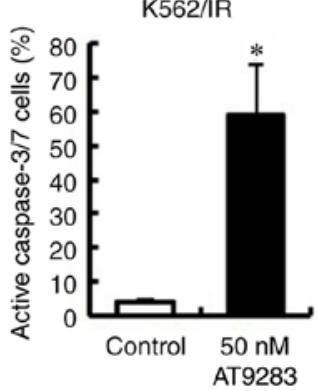

Figure 2. AT9283 increases the G2/M phase cell population and induces apoptosis of TKI-sensitive and TKI-resistant CML cells. K562 and K562/IR cell lines were treated with $50 \mathrm{nM}$ AT9283 for 2 days. (A) Assessment of the cell cycle analysis was performed with the Muse ${ }^{\mathrm{TM}}$ cell cycle kit. (B) Assessment of cells undergoing apoptosis was performed using the Muse ${ }^{\mathrm{TM}}$ Annexin- $\mathrm{V}$ and Dead Cell Assay kit. (C) Assessment of cells undergoing caspase-3/7 activation was performed with the Muse ${ }^{\mathrm{TM}}$ Caspase-3/7 kit. The results are expressed as the mean $\pm \mathrm{SD}$ of 3 experiments performed in triplicate. "P<0.05 compared to the control. TKI, tyrosine kinase inhibitor; CML, chronic myeloid leukemia.

\section{Results}

AT9283 decreases the cell viability of TKI-sensitive and TKI-resistant CML cells. We first investigated the effect of AT9283 on the cell viability using the trypan blue assay. We used the human CML cell line K562 and TKI-resistance cell line K562/IR. In our previous study, we showed that K562/IR exhibits resistance to TKIs including imatinib, nilotinib, dasatinib, bafetinib, and ponatinib (20). These cells were treated with AT9283 (10, 30, 50, and $100 \mathrm{nM})$ for 3 days. We found that AT9283 decreased the cell viability of both K562 (Fig. 1A) and K562/IR (Fig. 1B) cell lines in a concentration-dependent 
A

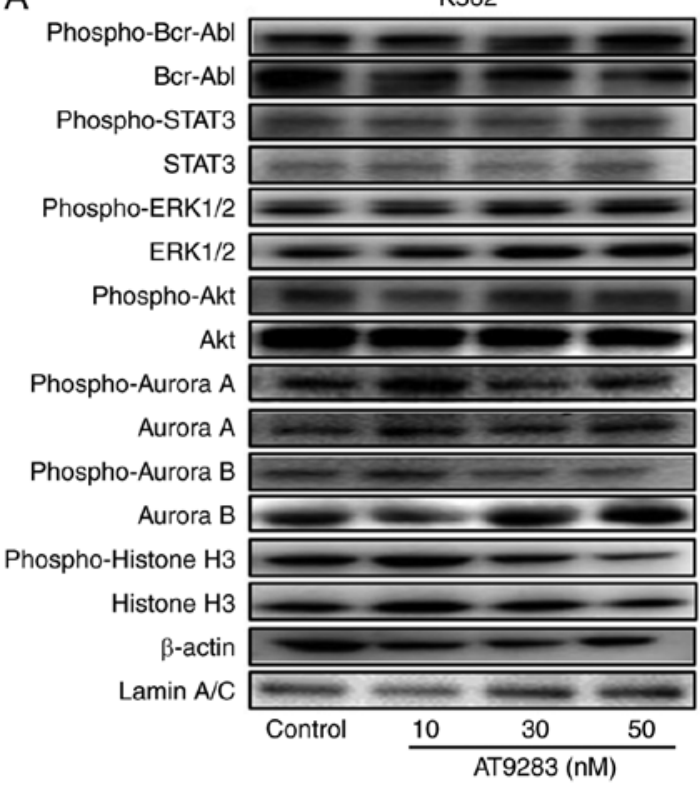

B
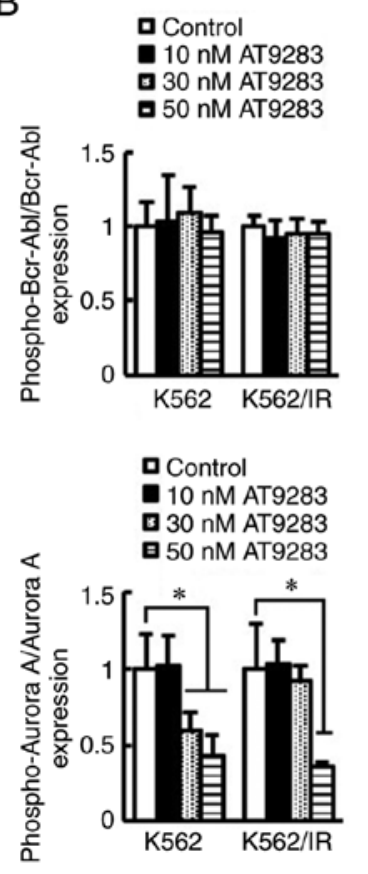
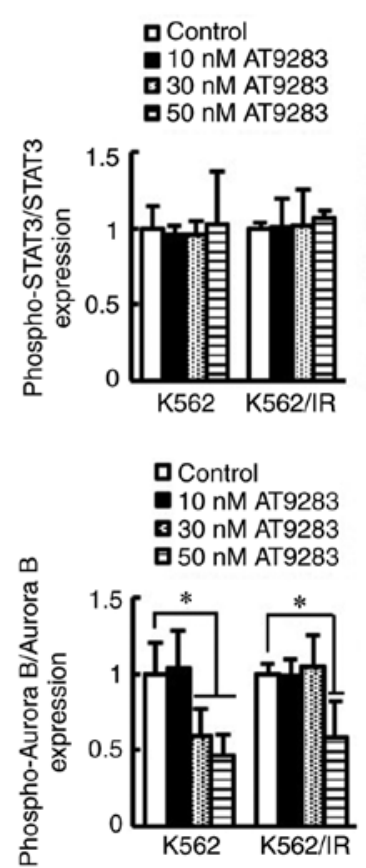
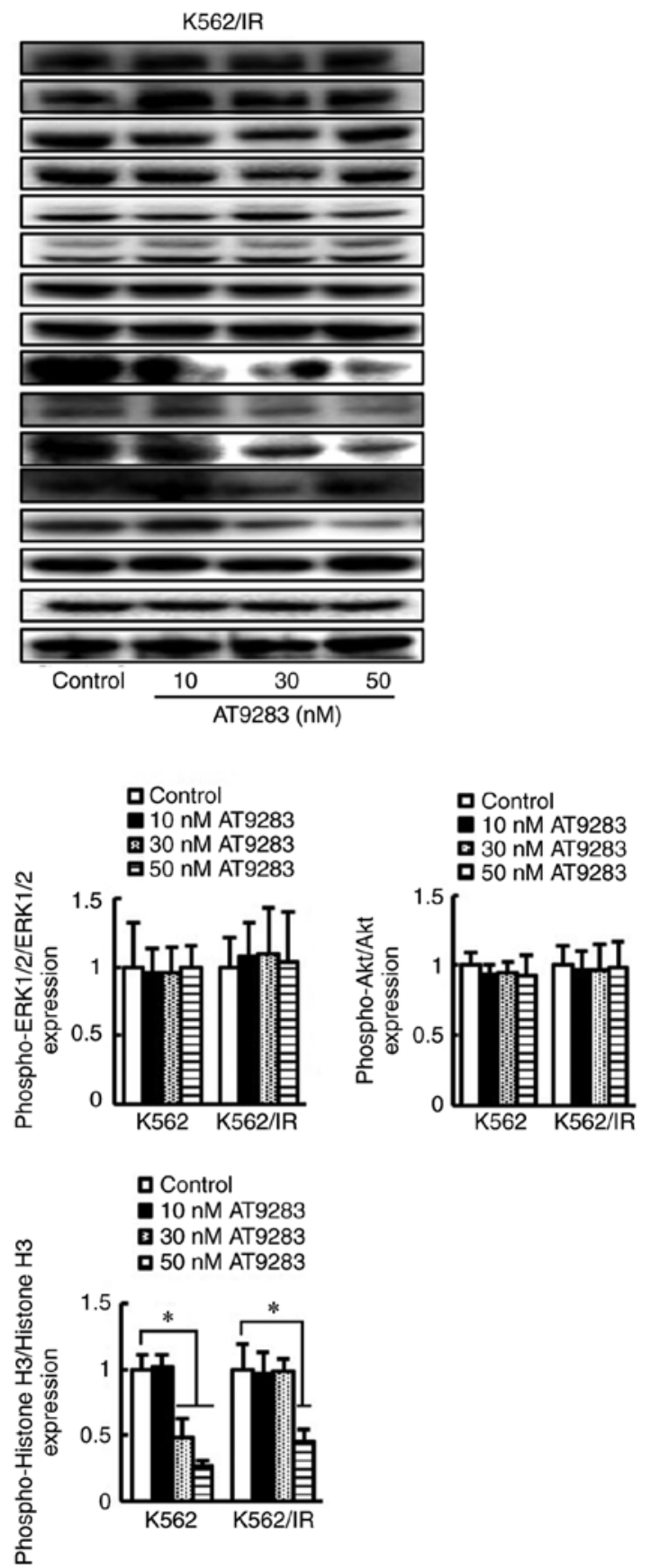

Figure 3. AT9283 suppresses Histone H3 phosphorylation via the inhibition of Aurora A and Aurora B in TKI-sensitive and TKI-resistant CML cells. (A) K562 and K562/IR cells were treated with AT9283 (10, 30, and $50 \mathrm{nM}$ ) for 2 days. The expression levels of phospho-Bcr-Abl, Bcr-Abl, phospho-STAT3, STAT3, phospho-ERK1/2, ERK1/2, phospho-Akt, Akt, phospho-Aurora A, Aurora A, phospho-Aurora B, Aurora B, phospho-Histone H3, and Histone H3 were detected using western blotting. The expression levels of $\beta$-actin and Lamin A/C were used as internal controls. (B) Quantification of signals is presented as fold change relative to phosphorylated protein vs. total protein. The results are representative of three independent experiments. ${ }^{*} \mathrm{P}<0.05$. TKI, tyrosine kinase inhibitor; CML, chronic myeloid leukemia; STAT3, signal transducer and activator of transcription 3; ERK, extracellular signal-regulated kinase.

manner. These results suggest that AT9283 decreases the cell viability of both TKI-sensitive and TKI-resistant CML cells.

AT9283 increases the cell population in the G2/M phase and induces apoptosis of TKI-sensitive and TKI-resistant CML cells. AT9283 has been reported to arrest the G2/M phase in tumor cells, ultimately leading to cell death by apoptosis (18). Therefore, we confirmed the effects of AT9283 on the cell cycle profile and the induction of apoptosis of CML cells (K562 and K562/IR). CML cells were treated with 50 nM AT9283 for 2 days, and the cell cycle profile was determined using the Muse $^{\mathrm{TM}}$ cell cycle kit. As expected, AT9283-treated CML cells (K562 and K562/IR) showed an increase in the G2/M phase (Fig. 2A). To further examine whether AT9283 induced apoptosis in CML cells, these cells were treated with 50 nM AT9283 for 2 days, and the number of apoptotic cells was measured using the Muse Annexin V and Dead Cell Assay kit. Our results showed that AT9283 treatment increased the number of total apoptotic cells in both K562 cells and K562/IR cell lines (Fig. 2B). Apoptosis is induced by an interaction between 
A

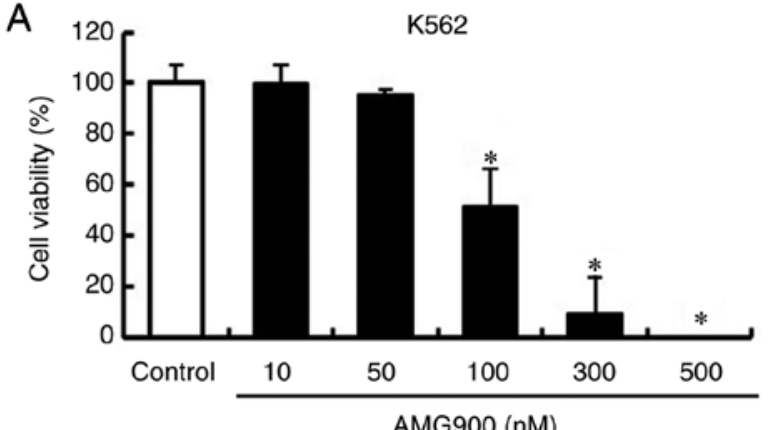

B

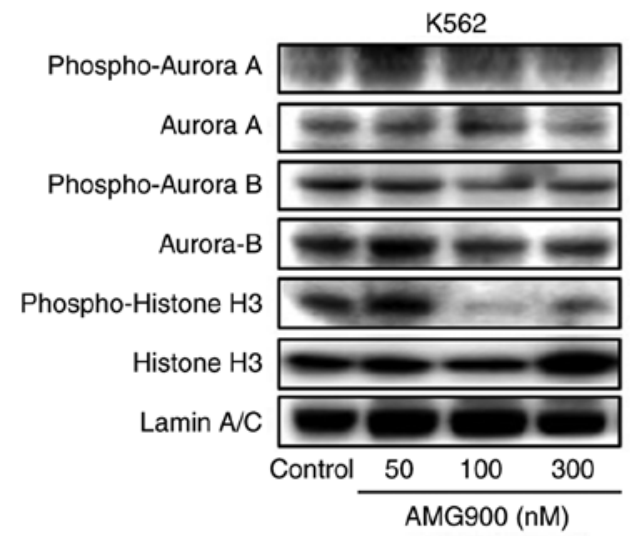

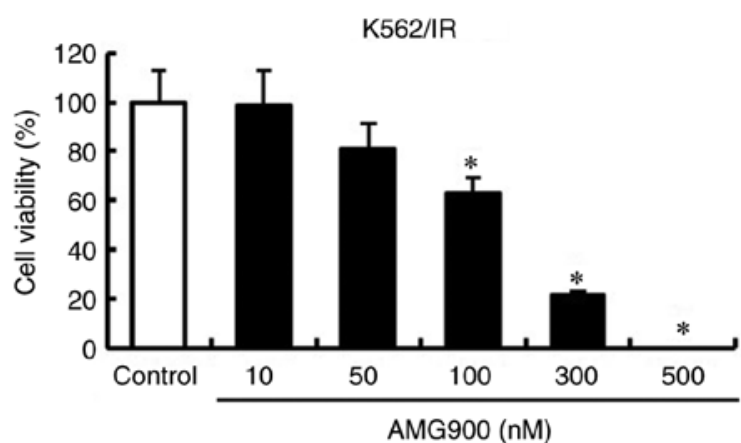

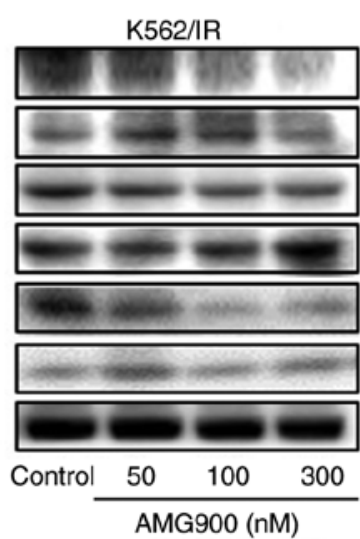

C
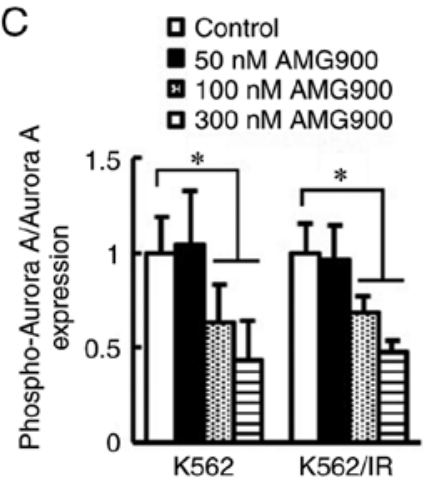
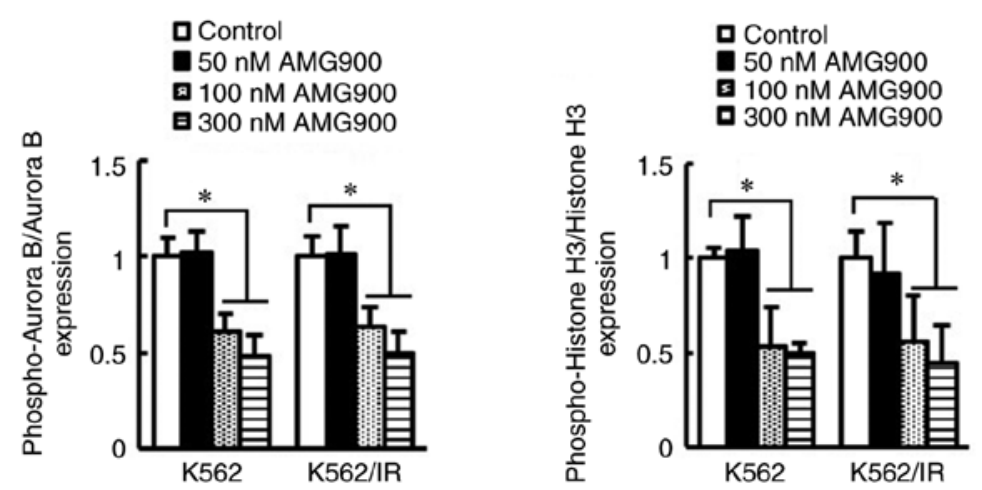

Figure 4. AMG900 decreases the cell viability via the inhibition of Aurora A and Aurora B of TKI-sensitive and TKI-resistant CML cells. (A) K562 and K562/IR cells were treated with AMG900 (10, 50, 100, 300, and $500 \mathrm{nM})$. Then, trypan blue exclusion assay was performed on the cells after 3 days. The results are expressed as mean $\pm \mathrm{SD}$ of 3 experiments performed in triplicate. ${ }^{*} \mathrm{P}<0.05$ compared to the control. (B) K562 and K562/IR cells were treated with AMG900 (50, 100, and $300 \mathrm{nM})$ for 2 days. The expression levels of phospho-Aurora A, Aurora A, phospho-Aurora B, Aurora B, phospho-Histone H3, and Histone $\mathrm{H} 3$ were detected using western blotting. The expression levels of Lamin A/C were used as internal controls. (C) Quantification of signals is presented as fold change relative to phosphorylated protein vs. total protein. The results are representative of three independent experiments. $\mathrm{P}<0.05$. TKI, tyrosine kinase inhibitor; CML, chronic myeloid leukemia.

various initiator and effector caspases $(22,23)$. Therefore, we confirmed the effects of AT9283 on caspase-3/7 activity in CML cells (K562 and K562/IR). CML cells were treated with $50 \mathrm{nM}$ AT9283 for 2 days, and the caspase-3/7 activity was determined using the Muse ${ }^{\text {TM }}$ Caspase-3/7 kit. We showed that AT9283 increased caspase-3/7 activity in both K562 cells and K562/IR cell lines (Fig. 2C). These results indicated that the decreased cell viability upon treatment with AT9283 is attributed to an increase in the $\mathrm{G} 2 / \mathrm{M}$ phase and induction of apoptosis.

AT9283 suppresses Histone H3 phosphorylation via inhibition of Aurora A and Aurora B in TKI-sensitive and TKI-resistant CML cells. AT9283 is a multi-targeted kinase inhibitor such as Abl, Aurora kinases, and JAK (18). To clarify the molecular mechanisms underlying the decrease in cell viability upon treatment with AT9283, we investigated the expression of phosphorylated Bcr-Abl, Aurora A, Aurora B, downstream STAT3, ERK, Akt, and Histone H3 in CML cells with AT9283 (10, 30, and $50 \mathrm{nM})$ by western blotting. Interestingly, our results showed that AT9283 significantly decreased the expression of Aurora A, Aurora B, and Histone $\mathrm{H} 3$ phosphorylation in both $\mathrm{K} 562$ cells and K562/IR cell lines, whereas we observed no changes in the levels of Bcr-Abl, STAT3, ERK, and Akt phosphorylation (Fig. 3). These results suggest that the decreased cell viability upon treatment with AT9283 may be involved in the inhibition of Histone $\mathrm{H} 3$ by decreasing the expression of phosphorylated Aurora A and Aurora B in both TKI-sensitive and TKI-resistant CML cells.

AMG900 increases G2/M phase and induces apoptosis via inhibition of Aurora A and Aurora B of TKI-sensitive and 

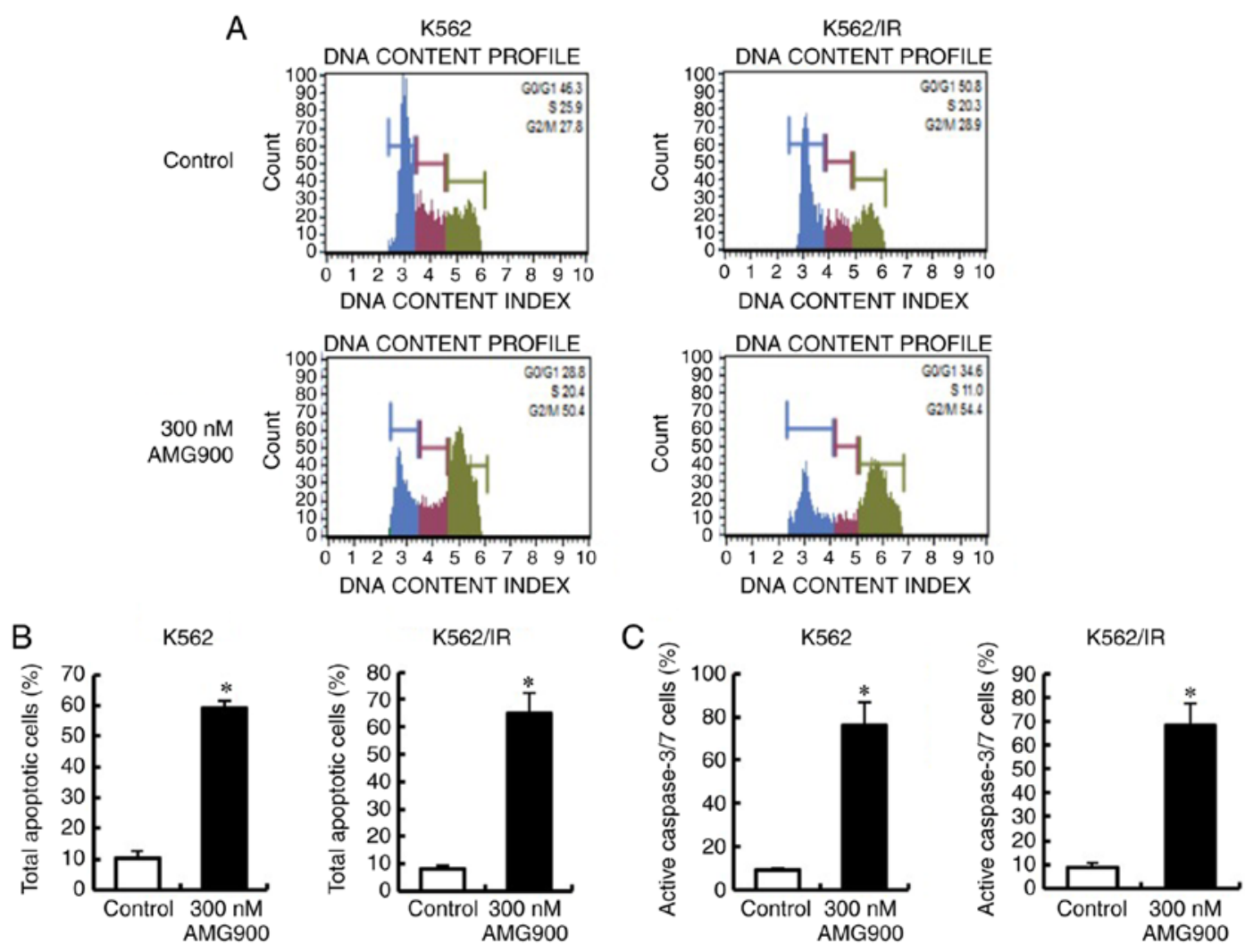

Figure 5. AMG900 increases the G2/M phase cell population and apoptosis of TKI-sensitive and TKI-resistant CML cells. K562 and K562/IR cells were treated with $300 \mathrm{nM}$ AMG900 for 2 days. (A) Assessment of the cell cycle analysis was performed with the Muse ${ }^{\mathrm{TM}}$ cell cycle kit. (B) Assessment of cells undergoing apoptosis was performed using the Muse ${ }^{\mathrm{TM}}$ Annexin-V and Dead Cell Assay kit. (C) Assessment of cells undergoing caspase-3/7 activation was performed with the Muse ${ }^{\mathrm{TM}}$ Caspase-3/7 kit. The results are expressed as the mean $\pm \mathrm{SD}$ of 3 experiments performed in triplicate. ${ }^{*} \mathrm{P}<0.05$ compared to the control. TKI, tyrosine kinase inhibitor; CML, chronic myeloid leukemia.

TKI-resistant CML cells. We further examined the role of Aurora A and Aurora B in the anticancer activity of AT9283 by using a selective AuroraA/B inhibitor AMG900. We showed that AMG900 decreased the cell viability and the expression of Aurora A, Aurora B, and Histone H3 phosphorylation in both K562 cells and K562/IR cell lines (Fig. 4). We also observed that AMG900 increased the G2/M phase population and significantly induced apoptosis and caspase $3 / 7$ activity (Fig. 5). These results support the critical role of Aurora A and Aurora B in the antiproliferative effects of AT9283 in both TKI-sensitive and TKI-resistant CML cells.

\section{Discussion}

In the present study, we investigated the effects of AT9283, a multi-targeted kinase inhibitor, in tyrosine kinase inhibitor (TKI)-sensitive chronic myeloid leukemia (CML) cells (K562) and TKI-resistant CML cells (K562/IR). We showed that AT9283 decreased the cell viability of both TKI-sensitive and TKI-resistant CML cells. In addition, we found that AT9283 increased the G2/M phase population and cell death by apoptosis. Preclinical studies have shown that AT9283 has potent cytotoxic and cell growth inhibitory activity in vitro as well as antitumor activity in vivo in a mouse xenograft model of colon carcinoma $(18,19)$. Furthermore, AT9283 has been reported to increase the G2/M phase and induce apoptosis of multiple myeloma and B-cell lymphoma cells in a time-dependent manner $(19,24)$. These results suggest that AT9283 may have potential clinical applications for the treatment of TKI-sensitive and TKI-resistant CML patients.

Differential sensitivity of AT9283 between K562 and K562/IR was observed. In our previous study, we showed that activation of MET in K562/IR cells was increased compared to K562 cells (20). MET is an oncogene encoding tyrosine kinase receptor, and plays an important role in embryogenesis, tumor growth, and metastasis (25). In addition, MET activation was reported to be associated with poorer response to chemotherapy. Therefore, differential sensitivity of AT9283 between K562 and K562/IR cell lines may involve MET activation. Further research will be carried out to investigate the differential sensitivity of AT9283 between K562 and K562/IR cells in the future.

AT9283 is a multi-targeted kinase inhibitor against Abl, Aurora kinases, and JAK (18). However, the molecular mechanisms underlying the decrease in cell viability of TKI-sensitive and TKI-resistant CML cells upon treatment with AT9283 was not unclear. Interestingly, our results showed that AT9283 decreased the expression of Aurora A, Aurora B, and Histone $\mathrm{H} 3$ phosphorylation. In contrast, we observed no changes in the levels of Bcr-Abl, STAT3, ERK, and Akt phosphorylation. Furthermore, we showed that AMG900, a selective Aurora A and Aurora B inhibitor, increased the G2/M phase cell population and induced apoptosis via the inhibition of Aurora A and Aurora B of both TKI-sensitive and TKI-resistant CML 
cells. Aurora A localizes to centrosomes during the G2 phase and is required for the separation and maturation of centrosomes through the recruitment of key components of spindle assembly (26). Aurora B kinase is also a chromosomal passenger protein that is essential for chromosome segregation through the phosphorylation of mitotic histone H3 $(27,28)$. Recent studies have reported that a decrease in Aurora A or Aurora B leads to G2/M arrest, spindle defects, and multi-nucleated cells, which eventually undergo apoptosis $(29,30)$. In addition, Aurora B kinase RNAi was evidenced by the inhibition of Histone $\mathrm{H} 3$ phosphorylation and endoreduplication, which leads to apoptosis in colon cancer cells (31). These results support the critical role of Aurora A and Aurora B in the antiproliferative effects of AT9283 in TKI-sensitive and TKI-resistant CML cells.

Differential cell viability between AT9283 and AMG900 in K562 and K562/IR was observed. In previous studies, AT9283 inhibited Aurora kinase at low concentration compared with AMG900 (18,32). Furthermore, our results showed that 30-50 nM of AT9283 inhibited the expression of Aurora A and Aurora B phosphorylation, whereas 100-300 nM of AMG900 inhibited the expression of Aurora A and Aurora B phosphorylation. Therefore, AT9283 may inhibit Aurora A and Aurora B more strongly than AMG900.

Different markers have been reported to predict the efficacy of Aurora inhibitors such as DNA aneuploidy, mitotic index, and percentage of aligned spindles in $\mathrm{G} 2 / \mathrm{M}$ phase $(33,34)$. In normal cells, Aurora A and Aurora B are expressed in a cell cycle-dependent manner, peaking during the $\mathrm{G} 2 / \mathrm{M}$ phase (35). Several transcription factors, such as E2F-1, E2F-4, DP-2 and FoxM1 have been reported to be implicated in cell cycle-regulated transcription of Aurora A and Aurora B (36-38). However, limited information is available concerning regulation of Aurora A and Aurora B expression in malignant tumors. Therefore, identifying the regulator of Aurora A and Aurora B will be an important task to clarify possible biomarkers of AT9283 in CML treatment.

Although the development of imatinib has led to an extended lifespan for many CML patients, the development of imatinib resistance represents a relevant clinical issue in the treatment of CML (39). To overcome this resistance, second-generation TKIs (nilotinib, dasatinib, and bosutinib) have been developed and are effective against a range of Bcr-Abl mutations (e.g., E255K, M351T), except for T315I (40,41). In addition, third-generation TKI ponatinib has activity against Bcr-Abl mutations, including T315I (42).

Although these drugs provide many benefits for patients with CML, in $50 \%$ of imatinib-resistant CML patients, there is no mutation in Bcr-Abl. Furthermore, the treatment of CML patients with Bcr-Abl-independent imatinib resistance has been disappointing. In the present study, we used K562/IR cells which did not have any Bcr-Abl mutations (20). Interestingly, our results showed that AT9283 decreased the cell viability of K562/IR cells in a concentration-dependent manner. Furthermore, AT9283 inhibited the expression of Aurora A, Aurora B, and downstream Histone H3 phosphorylation in K562/IR cells. Thus, our research provides new insights that Aurora A and Aurora B are promising therapeutic targets in Bcr-Abl-independent imatinib resistance, and AT9283 may have potential clinical application for the treatment of TKI-resistant CML patients with Bcr-Abl-independent imatinib resistance.
In conclusion, AT9283 decreased the cell viability of both TKI-sensitive and TKI-resistant CML cells. The decrease in cell viability was attributed to an increase in the G2/M phase population and induction of apoptosis via inhibition of Aurora A and Aurora B. Our research suggests that Aurora A and Aurora B are promising therapeutic targets in TKI-sensitive and TKI-resistant CML, and AT9283 may have potential clinical applications for CML treatment.

\section{Acknowledgements}

Not applicable.

\section{Funding}

This study was supported in part by a Grant-in-Aid for Young Scientists from the Japan Society for the Promotion of Science (JSPS).

\section{Availability of data and materials}

The datasets used during the present study are available from the corresponding author upon reasonable request.

\section{Authors' contributions}

TT performed the analysis of the trypan blue exclusion assay, cell cycle analysis, and western blotting and drafted the manuscript. MT, SG, CN and YO carried out analysis of the trypan blue exclusion assay, Annexin V assay, caspase-3/7 activity assay, and western blotting. MI and TS contributed to the statistical analyses. SN designed the experiments and revised the manuscript. All authors read and approved the manuscript and agree to be accountable for all aspects of the research in ensuring that the accuracy or integrity of any part of the work are appropriately investigated and resolved.

\section{Ethics approval and consent to participate}

Not applicable.

\section{Patient consent for publication}

Not applicable.

\section{Competing interests}

The authors declare that they have no competing interests.

\section{References}

1. Nie ZY, Yang L, Liu XJ, Yang Z, Yang GS, Zhou J, Qin Y, Yu J, Jiang LL, Wen JK, et al: Morin inhibits proliferation and induces apoptosis by modulating the miR-188-5p/PTEN/AKT regulatory pathway in CML cells. Mol Cancer Ther 18: 2296-2307, 2019.

2. Mukaida N, Tanabe Y and Baba T: Chemokines as a conductor of bone marrow microenvironment in chronic myeloid leukemia. Int J Mol Sci 18: 1824, 2017.

3. Chen SH, Chow JM, Hsieh YY, Lin CY, Hsu KW, Hsieh WS, Chi WM, Shabangu BM and Lee CH: HDAC1,2 knock-out and HDACi induced cell apoptosis in imatinib-resistant K562 cells. Int J Mol Sci 20: 2271, 2019. 
4. Chorzalska A, Ahsan N, Rao RSP, Roder K, Yu X, Morgan J, Tepper A, Hines S, Zhang P, Treaba DO, et al: Overexpression of Tpl2 is linked to imatinib resistance and activation of MEK-ERK and NF- $\mathrm{kB}$ pathways in a model of chronic myeloid leukemia. Mol Oncol 12: 630-647, 2018.

5. Bhamidipati PK, Kantarjian H, Cortes J, Cornelison AM and Jabbour E: Management of imatinib-resistant patients with chronic myeloid leukemia. Ther Adv Hematol 4: 103-117, 2013.

6. Sarno F, Pepe G, Termolino P, Carafa V, Massaro C, Merciai F Campiglia P, Nebbioso A and Altucci L: Trifolium Repens blocks proliferation in chronic myelogenous leukemia via the BCR-ABL/STAT5 pathway. Cells 9: 379, 2020.

7. Buffa $\mathrm{P}$, Romano $\mathrm{C}$, Pandini A, Massimino M, Tirrò E, Di Raimondo F, Manzella L, Fraternali F and Vigneri PG: BCR-ABL residues interacting with ponatinib are critical to preserve the tumorigenic potential of the oncoprotein. FASEB J 28 $1221-1236,2014$

8. Cimino G, Pane F, Elia L, Finolezzi E, Fazi P, Annino L Meloni G, Mancini M, Tedeschi A, Di Raimondo F, et al: The role of BCR/ABL isoforms in the presentation and outcome of patients with Philadelphia-positive acute lymphoblastic leukemia: A seven-year update of the GIMEMA 0496 trial Haematologica 91: 377-380, 2006.

9. Lin YG, Immaneni A, Merritt WM, Mangala LS, Kim SW, Shahzad MM, Tsang YT, Armaiz-Pena GN, Lu C, Kamat AA, et al: Targeting aurora kinase with MK-0457 inhibits ovarian cancer growth. Clin Cancer Res 14: 5437-5446, 2008.

10. Harrington EA, Bebbington D, Moore J, Rasmussen RK, Ajose-Adeogun AO, Nakayama T, Graham JA, Demur C, Hercend T, Diu-Hercend A, et al: VX-680, a potent and selective small-molecule inhibitor of the Aurora kinases, suppresses tumor growth in vivo. Nat Med 10: 262-267, 2004.

11. Matthews N, Visintin C, Hartzoulakis B, Jarvis A and Selwood DL: Aurora A and B kinases as targets for cancer: Will they be selective for tumors? Expert Rev Anticancer Ther 6: 109-120, 2006

12. Lapenna S and Giordano A: Cell cycle kinases as therapeutic targets for cancer. Nat Rev Drug Discov 8: 547-566, 2009.

13. Rodrigues Alves AP, Machado-Neto JA, Scheucher PS, Paiva HH, Simões BP, Rego EM and Traina F: Reversine triggers mitotic catastrophe and apoptosis in K562 cells. Leuk Res 48: 26-31, 2016

14. Gavriilidis P, Giakoustidis A and Giakoustidis D: Aurora kinases and potential medical applications of aurora kinase inhibitors: A review. J Clin Med Res 7: 742-751, 2015.

15. Ikezoe T, Yang J, Nishioka C, Tasaka T, Taniguchi A, Kuwayama Y, Komatsu N, Bandobashi K, Togitani K, Koeffler HP and Taguchi H A novel treatment strategy targeting Aurora kinases in acute myelogenous leukemia. Mol Cancer Ther 6: 1851-1857, 2007.

16. Tabe Y, Jin L, Iwabuchi K, Wang RY, Ichikawa N, Miida T, Cortes J, Andreeff $\mathrm{M}$ and Konopleva M: Role of stromal microenvironment in nonpharmacological resistance of CML to imatinib through Lyn/CXCR4 interactions in lipid rafts. Leukemia 26: 883-892, 2012.

17. Zhang X, Tu H, Yang Y, Jiang X, Hu X, Luo Q and Li J: Bone marrow-derived mesenchymal stromal cells promote resistance to tyrosine kinase inhibitors in chronic myeloid leukemia via the IL-7/JAK1/STAT5 pathway. J Biol Chem 294: 12167-12179, 2019.

18. Howard S, Berdini V, Boulstridge JA, Carr MG, Cross DM, Curry J, Devine LA, Early TR, Fazal L, Gill AL, et al: Fragment-based discovery of the pyrazol-4-yl urea (AT9283), a multitargeted kinase inhibitor with potent aurora kinase activity. J Med Chem 52: 379-388, 2009.

19. Qi W, Liu X, Cooke LS, Persky DO, Miller TP, Squires M and Mahadevan D: AT9283, a novel aurora kinase inhibitor, suppresses tumor growth in aggressive B-cell lymphomas. Int J Cancer 130: 2997-3005, 2012.

20. Tsubaki M, Takeda T, Kino T, Sakai K, Itoh T, Imano M, Nakayama T, Nishio K, Satou T and Nishida S: Contributions of MET activation to BCR-ABL1 tyrosine kinase inhibitor resistance in chronic myeloid leukemia cells. Oncotarget 8: 38717-38730, 2017.

21. Tsubaki M, Ogawa N, Takeda T, Sakamoto K, Shimaoka H, Fujita A, Itoh T, Imano M, Satou T and Nishida S: Dimethyl fumarate induces apoptosis of hematopoietic tumor cells via inhibition of NF- $\kappa B$ nuclear translocation and down-regulation of Bcl-xL and XIAP. Biomed Pharmacother 68: 999-1005, 2014

22. Tsubaki M, Fujiwara D, Takeda T, Kino T, Tomonari Y, Itoh T, Imano M, Satou T, Sakaguchi K and Nishida S: The sensitivity of head and neck carcinoma cells to statins is related to the expression of their Ras expression status, and statin-induced apoptosis is mediated via suppression of the Ras/ERK and Ras/mTOR pathways. Clin Exp Pharmacol Physiol 44: 222-234, 2017.
23. Takeda T, Tsubaki M, Tomonari Y, Kawashima K, Itoh T, Imano M, Satou T and Nishida S: Bavachin induces the apoptosis of multiple myeloma cell lines by inhibiting the activation of nuclear factor kappa B and signal transducer and activator of transcription 3. Biomed Pharmacother 100: 486-494, 2018.

24. Santo L, Hideshima T, Cirstea D, Bandi M, Nelson EA, Gorgun G, Rodig S, Vallet S, Pozzi S, Patel K, et al: Antimyeloma activity of a multitargeted kinase inhibitor, AT9283, via potent Aurora kinase and STAT3 inhibition either alone or in combination with lenalidomide. Clin Cancer Res 17: 3259-3271, 2011.

25. Liang $\mathrm{H}$ and Wang M: MET oncogene in non-small cell lung cancer: Mechanism of MET dysregulation and agents targeting the HGF/c-Met axis. Onco Targets Ther 13: 2491-2510, 2020

26. Hirota T, Kunitoku N, Sasayama T, Marumoto T, Zhang D, Nitta M, Hatakeyama K and Saya H: Aurora-A and an interacting activator, the LIM protein Ajuba, are required for mitotic commitment in human cells. Cell 114: 585-598, 2003.

27. Tao Y, Zhang P, Girdler F, Frascogna V, Castedo M, Bourhis J, Kroemer G and Deutsch E: Enhancement of radiation response in p53-deficient cancer cells by the Aurora-B kinase inhibitor AZD1152. Oncogene 27: 3244-3255, 2008.

28. Crosio C, Fimia GM, Loury R, Kimura M, Okano Y, Zhou H, Sen S, Allis CD and Sassone-Corsi P: Mitotic phosphorylation of histone H3: Spatio-temporal regulation by mammalian Aurora kinases. Mol Cell Biol 22: 874-885, 2002.

29. Marumoto T, Honda S, Hara T, Nitta M, Hirota T, Kohmura E and Saya H: Aurora-A kinase maintains the fidelity of early and late mitotic events in HeLa cells. J Biol Chem 278: 51786-51795, 2003

30. Giet R and Glover DM: Drosophila aurora B kinase is required for histone $\mathrm{H} 3$ phosphorylation and condensin recruitment during chromosome condensation and to organize the central spindle during cytokinesis. J Cell Biol 152: 669-682, 2001.

31. Curry J, Angove H, Fazal L, Lyons J, Reule M, Thompson N and Wallis N: Aurora B kinase inhibition in mitosis: Strategies for optimising the use of aurora kinase inhibitors such as AT9283. Cell Cycle 8: 1921-1929, 2009.

32. Payton M, Bush TL, Chung G, Ziegler B, Eden P, McElroy P, Ross S, Cee VJ, Deak HL, Hodous BL, et al: Preclinical evaluation of AMG 900, a novel potent and highly selective pan-aurora kinase inhibitor with activity in taxane-resistant tumor cell lines. Cancer Res 70: 9846-9854, 2010.

33. Chakravarty A, Shinde V, Tabernero J, Cervantes A, Cohen RB, Dees EC, Burris H, Infante JR, Macarulla T, Elez E, et al: Phase I assessment of new mechanism-based pharmacodynamic biomarkers for MLN8054, a small-molecule inhibitor of Aurora A kinase. Cancer Res 71: 675-685, 2011

34. Malumbres M and Pérez de Castro I: Aurora kinase A inhibitors: Promising agents in antitumoral therapy. Expert Opin Ther Targets 18: 1377-1393, 2014.

35. Zhang XH, Rao M, Loprieato JA, Hong JA, Zhao M, Chen GZ, Humphries AE, Nguyen DM, Trepel JB, Yu X and Schrump DS: Aurora A, Aurora B and survivin are novel targets of transcriptional regulation by histone deacetylase inhibitors in non-small cell lung cancer. Cancer Biol Ther 7: 1388-1397, 2009.

36. Vader G and Lens SM: The Aurora kinase family in cell division and cancer. Biochim Biophys Acta 1786: 60-72, 2008.

37. Tanaka M, Ueda A, Kanamori H, Ideguchi H, Yang J, Kitajima S and Ishigatsubo Y: Cell-cycle-dependent regulation of human aurora A transcription is mediated by periodic repression of E4TF1. J Biol Chem 277: 10719-10726, 2002.

38. Kimura M, Uchida C, Takano Y, Kitagawa M and Okano Y: Cell cycle-dependent regulation of the human aurora B promoter. Biochem Biophys Res Commun 316: 930-936, 2004.

39. Kantarjian HM, Talpaz M, Giles F, O'Brien S and Cortes J: New insights into the pathophysiology of chronic myeloid leukemia and imatinib resistance. Ann Intern Med 145: 913-923, 2006.

40. Zhou T, Commodore L, Huang WS, Wang Y, Thomas M, Keats J, Xu Q, Rivera VM, Shakespeare WC, Clackson T, et al: Structural mechanism of the Pan-BCR-ABL inhibitor ponatinib (AP24534): Lessons for overcoming kinase inhibitor resistance. Chem Biol Drug Des 77: 1-11, 2011.

41. Branford S, Melo JV and Hughes TP: Selecting optimal second-line tyrosine kinase inhibitor therapy for chronic myeloid leukemia patients after imatinib failure: Does the BCR-ABL mutation status really matter? Blood 114: 5426-5435, 2009.

42. Lan X, Zhao C, Chen X, Zhang P, Zang D, Wu J, Chen J, Long H, Yang L, Huang $\mathrm{H}$, et al: Platinum pyrithione induces apoptosis in chronic myeloid leukemia cells resistant to imatinib via DUB inhibition-dependent caspase activation and Bcr-Abl downregulation. Cell Death Dis 8: e2913, 2017. 